\title{
Weak compactness of operators acting on o-O type spaces
}

Article

Accepted Version

Perfekt, K.-M. (2015) Weak compactness of operators acting on o-O type spaces. Bulletin of the London Mathematical Society, 47 (4). pp. 677-685. ISSN 0024-6093 doi: https://doi.org/10.1112/blms/bdv031 Available at https://centaur.reading.ac.uk/71298/

It is advisable to refer to the publisher's version if you intend to cite from the work. See Guidance on citing.

Published version at: http://dx.doi.org/10.1112/b/ms/bdv031

To link to this article DOI: http://dx.doi.org/10.1112/blms/bdv031

Publisher: London Mathematical Society

All outputs in CentAUR are protected by Intellectual Property Rights law, including copyright law. Copyright and IPR is retained by the creators or other copyright holders. Terms and conditions for use of this material are defined in the End User Agreement.

\section{www.reading.ac.uk/centaur}

\section{CentAUR}

Central Archive at the University of Reading

Reading's research outputs online 


\title{
Weak compactness of operators acting on o-O type spaces
}

\author{
Karl-Mikael Perfekt
}

\begin{abstract}
We consider operators $T: M_{0} \rightarrow Z$ and $T: M \rightarrow Z$, where $Z$ is a Banach space and $\left(M_{0}, M\right)$ is a pair of Banach spaces belonging to a general construction in which $M$ is defined by a "big- $O$ " condition and $M_{0}$ is given by the corresponding "little-o" condition. Prototype examples of such spaces $M$ are given by $\ell^{\infty}$, weighted spaces of functions or their derivatives, bounded mean oscillation, Lipschitz-Hölder spaces, and many others. The main result characterizes the weakly compact operators $T$ in terms of a certain norm naturally attached to $M$, weaker than the $M$ norm, and shows that weakly compact operators $T: M_{0} \rightarrow Z$ are already quite close to being completely continuous. Further, we develop a method to extract $c_{0}$-subsequences from sequences in $M_{0}$. Applications are given to the characterizations of the weakly compact composition and Volterra-type integral operators on weighted spaces of analytic functions, BMOA, VMOA, and the Bloch space.
\end{abstract}

\section{Introduction}

Let $Z$ be a Banach space. The main result of this paper characterizes the weak compactness of operators $T: M_{0} \rightarrow Z$ and $T: M \rightarrow Z$, where $\left(M_{0}, M\right)$ is a pair of Banach spaces in which $M$ is defined by a "big- $O$ " condition and $M_{0}$ by the corresponding "little- $o$ " condition. See (2) and (3) for the precise definition. The class of spaces $\left(M_{0}, M\right)$ is large and examples include $c_{0}$ and $\ell^{\infty}$, weighted and the corresponding vanishing weighted spaces of continuous, analytic or harmonic functions, Möbius invariant spaces of analytic functions, Lipschitz-Hölder spaces, bounded and vanishing mean oscillation (BMO and VMO), and several others. The pair $\left(M_{0}, M\right)$ was first introduced in [14], and the quoted examples are given there.

This paper is inspired by recent works on the compactness properties of composition and integral operators acting on specific examples of spaces $M_{0}$ and $M[\mathbf{3}],[\mathbf{7}],[\mathbf{8}],[\mathbf{1 0}]$. It often turns out that weak compactness and compactness are equivalent for these classes of operators, a phenomenon which can be readily understood given the main results of this article.

For the statements of the theorems, note that $M$ is associated with a reflexive Banach space $X$ in which $M$ is continuously contained (see Section 2). For instance, $\ell^{\infty}$ is continuously contained in a weighted $\ell^{2}$-space.

Theorem 3.2. A bounded operator $T: M_{0} \rightarrow Z$ is weakly compact if and only if there for each $\varepsilon>0$ exists an $N>0$ such that

$$
\|T x\|_{Z} \leq N\|x\|_{X}+\varepsilon\|x\|_{M}, \quad x \in M_{0} .
$$

A similar description of the weakly compact operators on $C(K)$-spaces was given by Niculescu, and a generalization to operators acting on general $C^{*}$-algebras is due to Jarchow [6]. More recently, characterizations in the same spirit have been given for operators acting on $H^{\infty}([\mathbf{9}])$ and certain subspaces of Orlicz spaces [10].

2000 Mathematics Subject Classification 46B50 (primary), 30H30, 30H35, 46E15, 47A99, 47B33, 47G10 (secondary). 
In [14] it was proven that $M_{0}^{* *} \simeq M$ in a canonical way. Therefore, Theorem 3.2 also applies to operators $T: M \rightarrow Z$ such that $\left(\left.T\right|_{M_{0}}\right)^{* *}=T$ - i.e. operators $T$ which are weak ${ }^{*}$-weak continuous, a continuity property which is simple to verify in many concrete examples. See Corollary 3.3.

To compare the weak compactness characterization with compactness criteria, note that $T: M_{0} \rightarrow Z$ is completely continuous if and only if for every bounded sequence $\left(x_{n}\right) \subset M_{0}$ such that $x_{n}$ converges weakly to zero, it holds that $\lim _{n}\left\|T x_{n}\right\|_{Z}=0$. To demand instead only weak compactness, one simply replaces the weak convergence of $x_{n}$ with the stronger property (see [14]) that $x_{n}$ converges to zero in $X$-norm. The two conditions on the sequence $\left(x_{n}\right)$ are in many concrete examples closely related; herein lies the explanation of why weak compactness and compactness often are equivalent for operators on $M_{0}$ and $M$. See the examples in Section 4.

The motivation for the proof of Theorem 3.2 comes from [15], where it was shown that $M_{0}$ is an M-ideal in $M$. In particular, weakly compact operators on $M_{0}$ can be characterized in terms of $c_{0}$-subspaces of $M_{0}$. The proof hence relies on a procedure to create $c_{0}$-subspaces, a construction which we summarize as a separate theorem.

Theorem 3.1. Suppose that $x_{n} \in M_{0}, n=1,2,3, \ldots$, is a sequence such that $\left\|x_{n}\right\|_{M}=1$ and $\lim _{n \rightarrow \infty}\left\|x_{n}\right\|_{X}=0$. Then $\left(x_{n}\right)_{n}$ has a subsequence which, as a basic sequence in $M_{0}$, is equivalent to the canonical basis of $c_{0}$.

This result is classical for $M_{0}=c_{0}$, and has also been proven for the case when $M_{0}=\mathrm{VMO}$ $[\mathbf{1 2}]$, the latter fact which has been used in $[\mathbf{7}]$ and $[\mathbf{8}]$ to characterize the weak compactness of Volterra-type integral operators and composition operators on the analytic BMO-space.

The paper is organized as follows. In Section 2 the definitions of $M_{0}$ and $M$ are given, as well as technical preliminaries; in Section 3 the main results are proven; Section 4 gives applications of Theorem 3.2 and its corollary to composition and integral operators on weighted spaces of analytic functions, Bloch spaces, and analytic BMO-spaces.

\section{Definitions and preliminaries}

The spaces $M$ and $M_{0}$ are defined by

$$
M(X, \mathcal{L})=\left\{x \in X: \sup _{L \in \mathcal{L}}\|L x\|_{Y}<\infty\right\}
$$

and

$$
M_{0}(X, \mathcal{L})=\left\{x \in M(X, \mathcal{L}): \varlimsup_{\mathcal{L} \ni L \rightarrow \infty}\|L x\|_{Y}=0\right\}
$$

Here $X$ and $Y$ are Banach spaces, where $X$ is assumed to be separable and reflexive. $\mathcal{L}$ is a collection of continuous linear operators $L: X \rightarrow Y$ that is made into a topological space $(\mathcal{L}, \tau)$ by a $\sigma$-compact locally compact Hausdorff topology $\tau$. The topology should respect the strong operator topology in the sense that for every $x \in X$, the map $T_{x}: \mathcal{L} \rightarrow Y$ given by $T_{x} L=L x$ is continuous. The limit $L \rightarrow \infty$ in the definition of $M_{0}$ should be understood in the sense of one-point compactification of $(\mathcal{L}, \tau)$ (i.e. $L$ should escape all compact sets).

We may assume that $M(X, \mathcal{L})$ is dense in $X[\mathbf{1 4}]$, and we suppose that

$$
\|x\|_{M(X, \mathcal{L})}=\sup _{L \in \mathcal{L}}\|L x\|_{Y}
$$

defines a norm on $M(X, \mathcal{L})$ which is stronger than the $X$-norm. As in the concrete examples mentioned in the introduction, we want to consider the situation where the bidual $M_{0}^{* *}$ can 
be canonically identified with $M$. For this to be true it is necessary to impose the following approximation property.

Assumption A. For every $x \in M(X, \mathcal{L})$ there is a bounded sequence $\left\{x_{n}\right\}_{n=1}^{\infty}$ in $M_{0}(X, \mathcal{L})$ such that $x_{n}$ converges weakly to $x$ in $X$.

Henceforth we always assume that A holds. There is also the stronger hypothesis:

Assumption B. For every $x \in M(X, \mathcal{L})$ there is a bounded sequence $\left\{x_{n}\right\}_{n=1}^{\infty}$ in $M_{0}(X, \mathcal{L})$ such that $x_{n}$ converges weakly to $x$ in $X$ and $\sup _{n}\left\|x_{n}\right\|_{M(X, \mathcal{L})} \leq\|x\|_{M(X, \mathcal{L})}$.

The next theorem, stating that indeed $M_{0}^{* *}=M$ holds, was proven in [14]. For its statement, note that $M_{0}(X, \mathcal{L})$ can be viewed as a closed subspace of both $M$ and $M_{0}^{* *}$.

Theorem $2.1[\mathbf{1 4}]$. The dual space $X^{*}$ is continuously contained and dense in $M_{0}(X, \mathcal{L})^{*}$. Denoting by

$$
I: M_{0}(X, \mathcal{L})^{* *} \rightarrow X
$$

the adjoint of the inclusion map $J: X^{*} \rightarrow M_{0}(X, \mathcal{L})^{*}$, the operator $I$ is a continuous isomorphism of $M_{0}(X, \mathcal{L})^{* *}$ onto $M(X, \mathcal{L})$ which acts as the identity on $M_{0}(X, \mathcal{L})$. Furthermore, $I$ is an isometry if Assumption $B$ holds.

In the isometric case the author proved in [15] that $M_{0}$ is an $M$-ideal in $M$. In particular, $M_{0}$ has Pełczyński's property $(\mathrm{V})$, which as a consequence gives the following characterization of weakly compact operators on $M_{0}$ (see [5]).

Proposition 2.2 [15]. Suppose that Assumption $B$ holds. If $Z$ is a Banach space and $T: M_{0}(X, \mathcal{L}) \rightarrow Z$ is a bounded operator, then $T$ is weakly compact if and only if there does not exist a subspace $F \subset M_{0}(X, \mathcal{L})$ isomorphic to $c_{0}$ such that $\left.T\right|_{F}$ is an isomorphism.

The proof of Theorem 3.2 is inspired by this proposition, but technically only relies on its forward direction which follows easily for any Banach space from the fact that $c_{0}$ has the Dunford-Pettis property.

A sequence $\left(z_{n}\right)_{n=1}^{\infty}$ in a Banach space $Z$ is called basic if it is a (Schauder) basis for its span $\left[z_{n}\right]=\operatorname{span}\left\{z_{n}\right\}$. Two basic sequences $\left(z_{n}\right)$ and $\left(w_{n}\right)$ in Banach spaces $Z$ and $W$, respectively, are said to be equivalent if there is an isomorphism between $\left[z_{n}\right]$ and $\left[w_{n}\right]$ which maps $z_{n}$ onto $w_{n}$, for all $n$. In this situation, if $W=c_{0}$ and $\left(w_{n}\right)$ is the unit-vector basis of $c_{0}$, we say that $\left(z_{n}\right)$ is equivalent to the canonical basis of $c_{0}$. For rudimentary information about bases, we refer to the classical paper of Bessaga and Pełczyński [1], the techniques of which will be utilized to prove the main results of this paper.

\section{Results and Proofs}

In the proof of Theorem 3.1 we make use of the embedding operator $V: M_{0}(X, \mathcal{L}) \rightarrow$ $C_{0}(\mathcal{L}, Y)$ which isometrically embeds $M_{0}$ into the space of continuous $Y$-valued functions on 
$\mathcal{L}$ vanishing at infinity. Explicitly

$$
V x(L)=L x, \quad x \in M_{0}, L \in \mathcal{L} .
$$

Theorem 3.1. Suppose that $x_{n} \in M_{0}(X, \mathcal{L}), n=1,2,3, \ldots$, is a sequence such that $\left\|x_{n}\right\|_{M}=1$ and $\lim _{n \rightarrow \infty}\left\|x_{n}\right\|_{X}=0$. Then $\left(x_{n}\right)_{n}$ has a subsequence which, as a basic sequence in $M_{0}(X, \mathcal{L})$, is equivalent to the canonical basis of $c_{0}$.

Proof. We will construct a subsequence $\left(z_{n}\right)_{n}$ of $\left(x_{n}\right)_{n}$ inductively. We will also construct two auxiliary sequences; a strictly increasing sequence of positive integers $\left(\beta_{n}\right)_{n}$, and a sequence $\left(f_{n}\right)_{n}$ in $B(\mathcal{L}, Y)$, the space of bounded Baire measurable $Y$-valued functions equipped with the supremum norm. To begin, let $z_{1}=x_{1}, \beta_{1}=1$ and $f_{1}=V z_{1}$. For the construction, fix a strictly increasing sequence $\mathcal{K}_{1} \subset \mathcal{K}_{2} \subset \cdots$ of compact Baire subsets of $(\mathcal{L}, \tau)$ such that $\mathcal{L}=\bigcup_{n=1}^{\infty} \mathcal{K}_{n}$. We denote by $\mathcal{K}_{n}^{c}$ the complement of $\mathcal{K}_{n}$ in $\mathcal{L}$.

Suppose now that $z_{1}, \ldots, z_{n-1}, \beta_{1}, \ldots, \beta_{n-1}$, and $f_{1}, \ldots, f_{n-1}$ have been chosen. Since each $z_{j}$ belongs to $M_{0}(X, \mathcal{L})$ we can pick $\beta_{n}>\beta_{n-1}$ such that

$$
\left\|L z_{j}\right\|_{Y} \leq 1 / 2^{j}, \quad L \in \mathcal{K}_{\beta_{n}}^{c}, j=1, \ldots, n-1 .
$$

Since the operators $L \in \mathcal{K}_{\beta_{n}}$ are uniformly bounded by the Banach-Steinhaus theorem, it follows from $\lim _{k}\left\|x_{k}\right\|_{X}=0$ that we may choose $z_{n}$ to be an element from $\left(x_{k}\right)_{k}$ such that

$$
\left\{L \in \mathcal{L}:\left\|L z_{n}\right\|_{Y}>1 / 2^{n}\right\} \subset \mathcal{K}_{\beta_{n}}^{c} .
$$

Denoting the set on the left hand side of (5) by $\mathcal{A}_{n}$, let

$$
f_{n}=\mathbb{1}_{\mathcal{A}_{n}} V z_{n}
$$

where $\mathbb{1}_{\mathcal{A}_{n}}$ is the characteristic function of $\mathcal{A}_{n}$.

With the inductive process complete, we now claim that $\left(z_{n}\right)_{n=2}^{\infty} \subset M_{0}(X, \mathcal{L})$ has a further subsequence equivalent to the canonical basis of $c_{0}$. To see this, let

$$
\mathcal{B}_{n}=\mathcal{A}_{n} \backslash \cup_{j>n} \mathcal{A}_{j}, \quad n \geq 2 .
$$

If $L \in \mathcal{B}_{m}$ for some $m \geq 2$, then $f_{n}(L)=0$ for $n>m$, while by construction

$$
\left\|f_{n}(L)\right\|_{Y} \leq 1 / 2^{n} \text {, for } n<m .
$$

Since $\left\|x_{k}\right\|_{M}=1$ for all $k$, we of course have that $\left\|f_{m}(L)\right\|_{Y} \leq 1$. Hence, for $L \in \mathcal{B}_{m}$ we have

$$
\sum_{n=2}^{\infty}\left\|f_{n}(L)\right\|_{Y} \leq 1+\sum_{n=2}^{m-1} \frac{1}{2^{n}}<3 / 2 .
$$

On the other hand, if $L \in\left(\cup_{k} \mathcal{B}_{k}\right)^{c}$, then $f_{n}(L)=0$ for every $n \geq 2$, since $\cup_{k} \mathcal{B}_{k}=\cup_{k} \mathcal{A}_{k}$. For the latter equality, note that no $L \in \mathcal{L}$ can belong to infinitely many sets $\mathcal{A}_{k}$, since $\mathcal{A}_{k} \subset \mathcal{K}_{\beta_{k}}^{c}$.

We have hence shown that

$$
\sum_{n=2}^{\infty}\left\|f_{n}(L)\right\|_{Y}<3 / 2, \quad \forall L \in \mathcal{L}
$$

Therefore, for any bounded sequence of numbers $\left(t_{n}\right)_{n}$, we find that

$$
\sup _{k}\left\|\sum_{n=2}^{k} t_{n} f_{n}(L)\right\|_{Y}<\frac{3}{2} \sup _{n}\left|t_{n}\right|, \quad \forall L \in \mathcal{L} .
$$

This latter inequality implies that the series $\sum_{n=2}^{\infty} f_{n}$ is weakly unconditionally Cauchy in $B(\mathcal{L}, Y)$ (see $[4]$, p. 44 ). Note also that each $f_{n}$ was constructed as to have supremum norm 1 , $\left\|f_{n}\right\|_{\infty}=1$. By the Bessaga-Pełczyński selection principle (C. 1. and Lemma 3 of $[\mathbf{1}]$ ) there is 
hence a basic subsequence $\left(f_{n_{k}}\right)_{k}$ equivalent to the canonical basis of $c_{0}$. But then there is a positive integer $K$ such that $\left(V z_{n_{k}}\right)_{k \geq K}$ is also basic and equivalent to the canonical basis of $c_{0}$, since

$$
\sum_{k=1}^{\infty}\left\|V z_{n_{k}}-f_{n_{k}}\right\|_{\infty} \leq \sum_{k=1}^{\infty} \frac{1}{2^{n_{k}}}<1
$$

This proves that $\left(z_{n_{k}}\right)_{k \geq K}$ is a subsequence of the desired type.

Based on Theorem 3.1 we now prove Theorem 3.2.

Theorem 3.2. Let $Z$ be a Banach space. A bounded operator $T: M_{0}(X, \mathcal{L}) \rightarrow Z$ is weakly compact if and only if there for each $\varepsilon>0$ exists an $N>0$ such that

$$
\|T x\|_{Z} \leq N\|x\|_{X}+\varepsilon\|x\|_{M}, \quad x \in M_{0}(X, \mathcal{L}) .
$$

Proof. Since $X$ is reflexive, the inclusion $j: M_{0}(X) \rightarrow X$ is a weakly compact map. Based on this observation, it is a relatively well known fact that having (6) implies the weak compactness of $T$ (see e.g. Proposition 10 in [10]).

In the converse direction, suppose that (6) does not hold. Equivalently, there is an $\varepsilon>0$ and a sequence $\left(x_{n}\right)_{n} \subset M_{0}(X, \mathcal{L})$ with $\left\|x_{n}\right\|_{M}=1$ such that

$$
\left\|T x_{n}\right\|_{Z}>n\left\|x_{n}\right\|_{X}+\varepsilon \text {. }
$$

The boundedness of $T$ then automatically imposes $\lim _{n}\left\|x_{n}\right\|_{X}=0$. Therefore Theorem 3.1 applies, so that by passing to a subsequence we may assume that $\left(x_{n}\right) \subset M_{0}$ is equivalent to the canonical basis of $c_{0}$. In particular $\sum_{n} x_{n}$ is weakly unconditionally Cauchy in $M_{0}$, and hence $\sum_{n} T x_{n}$ is weakly unconditionally Cauchy in $Z$. Since also $\left\|T x_{n}\right\|_{Z} \geq \varepsilon$ for all $n$, there is, by the Bessaga-Pełczyński selection principle, a further subsequence $\left(T x_{n_{k}}\right)$ which too is equivalent to the canonical basis of $c_{0}$. But then both $\left(x_{n_{k}}\right)$ and $\left(T x_{n_{k}}\right)$ are equivalent to the canonical basis of $c_{0}$, and $T$ must act as an isomorphism between the two $c_{0}$-subspaces $\left[x_{n_{k}}\right] \subset M_{0}$ and $\left[T x_{n_{k}}\right] \subset Z$. Hence $T$ could not be weakly compact, or the Dunford-Pettis property of $c_{0}$ would be violated.

As a corollary of Theorem 3.2 we obtain the corresponding result for operators $T$ : $M(X, \mathcal{L}) \rightarrow Z$ which are weak* ${ }^{*}$-weak continuous. The weak ${ }^{*}$-topology of $M(X, \mathcal{L})$ referred to is the one induced by the duality in Theorem 2.1. Hence, letting $I$ denote the map of Theorem 2.1 and $T_{0}$ the restriction $T_{0}=\left.T\right|_{M_{0}}$, we have that weak ${ }^{*}$-weak continuity of $T$ means precisely that $T_{0}^{* *} I^{-1}=T$, which by abuse of notation typically is written as $T_{0}^{* *}=T$.

Corollary 3.3. Let $Z$ be a Banach space and $T: M(X, \mathcal{L}) \rightarrow Z$ be a bounded and weak*weak continuous operator. Then $T$ is weakly compact if and only if there for each $\varepsilon>0$ exists $N>0$ such that

$$
\|T x\|_{Z} \leq N\|x\|_{X}+\varepsilon\|x\|_{M}, \quad x \in M(X, \mathcal{L}) .
$$

Proof. Let $T_{0}=\left.T\right|_{M_{0}}$. The continuity hypothesis can equivalently be stated as $T_{0}^{* *}=T$. Hence it follows from Gantmacher's theorem that $T$ is weakly compact if and only if (6) holds.

It remains to see that (6) implies (7). Suppose that $\varepsilon, N>0$ are such that (6) holds and let $x \in M(X, \mathcal{L})$. We renorm $M_{0}^{* *}$ by equipping it with the equivalent norm

$$
\left\|I^{-1} x\right\|_{\text {alt }}=N\|x\|_{X}+\varepsilon\|x\|_{M}, \quad I^{-1} x \in M_{0}^{* *} .
$$


Invoking the weak-star-metrizability of the unit ball of $M_{0}^{* *}\left(M_{0}^{*}\right.$ is separable by Theorem 2.1), it follows that there exists a sequence of points $x_{n} \in M_{0}(X, \mathcal{L})$ converging weak-star to $x$ such that

$$
N\left\|x_{n}\right\|_{X}+\varepsilon\left\|x_{n}\right\|_{M} \leq N\|x\|_{X}+\varepsilon\|x\|_{M}, \quad \forall n .
$$

By the continuity of $T, T x_{n}$ converges weakly to $T x$, and therefore

$$
\|T x\|_{Z} \leq \frac{\lim }{n}\left\|T x_{n}\right\|_{Z} \leq \frac{\lim }{n}\left(N\left\|x_{n}\right\|_{X}+\varepsilon\left\|x_{n}\right\|_{M}\right) \leq N\|x\|_{X}+\varepsilon\|x\|_{M} .
$$

\section{Examples}

Our first example will be of a general nature, to illustrate the idea that when compactness for a class of operators can be determined through a testing condition, then Corollary 3.3 may sometimes be used to show that weak compactness and compactness are equivalent for the class.

EXAmPle 1. Suppose that $\left\{T_{\alpha}\right\}_{\alpha}$ is a family of bounded weak*-weak continuous operators $T_{\alpha}: M(X, \mathcal{L}) \rightarrow Z, Z$ a Banach space, and that there is a "testing sequence" $\left(x_{n}\right) \subset M(X, \mathcal{L})$ such that:

- the sequence $\left(x_{n}\right)$ is bounded in $M(X, \mathcal{L})$,

$-\lim _{n}\left\|x_{n}\right\|_{X}=0$, and

- for every $\alpha, \lim _{n}\left\|T_{\alpha} x_{n}\right\|_{Z}=0$ implies that $T_{\alpha}$ is compact.

Suppose now that $T_{\alpha}$ is weakly compact. Then Corollary 3.3 immediately implies that $T_{\alpha} x_{n}$ must tend to zero in $Z$, so that $T_{\alpha}$ is actually compact. Hence, in the above situation, an operator $T_{\alpha}$ is compact if and only if it is weakly compact if and only if $\lim _{n}\left\|T_{\alpha} x_{n}\right\|_{Z}=0$.

We now turn to several concrete examples of composition and integral operators acting on spaces of analytic functions. For an analytic function $\varphi: \mathbb{D} \rightarrow \mathbb{D}, C_{\varphi}$ denotes the composition operator

$$
C_{\varphi} f(z)=f(\varphi(z)), \quad z \in \mathbb{D},
$$

where $f$ is a holomorphic function on $\mathbb{D}, f \in \operatorname{Hol}(\mathbb{D})$. We begin by considering composition operators $C_{\varphi}$ on weighted spaces.

EXAmple 2. Let $v: \mathbb{D} \rightarrow \mathbb{R}_{+}$be a strictly positive, radial, continuous weight on $\mathbb{D}$ such that $\lim _{|z| \rightarrow 1} v(z)=0$, and consider the weighted spaces of holomorphic functions

$$
H_{v}^{\infty}=\left\{f \in \operatorname{Hol}(\mathbb{D}): \sup _{z \in \mathbb{D}}|f(z)| v(z)<\infty\right\}
$$

and

$$
H_{v}^{0}=\left\{f \in \operatorname{Hol}(\mathbb{D}): \varlimsup_{|z| \rightarrow 1^{-}}|f(z)| v(z)=0\right\} .
$$

They can be realized within our framework ([14], Example 4.4) with the role of $X$ taken on by the analytic Bergman space on the disc with weight $v^{2}$;

$$
X=L_{a}^{2}\left(v^{2} d A, \mathbb{D}\right)=L^{2}\left(v^{2} d A, \mathbb{D}\right) \cap \operatorname{Hol}(\mathbb{D}),
$$

where $d A=d x d y$ denotes area measure. $H_{v}^{\infty}$ and $H_{v}^{0}$ are obtained by letting $Y=\mathbb{C}$ and letting $\mathcal{L}$ consist of the linear functionals $L_{z}, z \in \mathbb{D}$, defined by $L_{z} f=|v(z)| f(z)$. The topology we 
impose on $\mathcal{L}$ is simply the one inherited from $\mathbb{D}$ through the parametrization $z \mapsto L_{z}$. The desired approximation property Assumption B can be verified by considering dilations $f(r z)$ of a function $f \in H_{v}^{\infty}, r<1$ (see [2]).

Let

$$
\tilde{u}(z)=\sup _{\|f\|_{H_{v}^{\infty}} \leq 1}|f(z)|
$$

and associate with $v$ the weight $\tilde{v}=1 / \tilde{u}$. Then $\tilde{v}$ is a weight of the same type as $v$ and $H_{v}^{\infty}=H_{\tilde{v}}^{\infty}$ isometrically $([\mathbf{2}]) . v$ is called essential if $v$ is comparable to $\tilde{v}$. Given also a weight $w$ of the same type as $v$, Bonet et. al. characterized in [2] the compact composition operators $C_{\varphi}: H_{v}^{\infty} \rightarrow H_{w}^{\infty}$. We utilize Theorem 3.2 to add also weak compactness to their description. For simplicity we suppose that both $v$ and $w$ are essential.

Proposition 4.1. The following are equivalent:

i) $C_{\varphi}: H_{v}^{\infty} \rightarrow H_{w}^{\infty}$ is compact,

ii) $C_{\varphi}: H_{v}^{0} \rightarrow H_{w}^{0}$ is compact,

iii) $\lim _{r \rightarrow 1^{-}} \sup _{|\varphi(z)|>r} \frac{w(z)}{v(\varphi(z))}=0$ or $\overline{\varphi(\mathbb{D})} \subset \mathbb{D}$,

iv) $\lim _{|z| \rightarrow 1^{-}} \frac{w(z)}{v(\varphi(z))}=0$,

i') $C_{\varphi}: H_{v}^{\infty} \rightarrow H_{w}^{\infty}$ is weakly compact,

ii') $C_{\varphi}: H_{v}^{0} \rightarrow H_{w}^{0}$ is weakly compact.

iii') $C_{\varphi}\left(H_{v}^{\infty}\right) \subset H_{w}^{0}$

Proof. The equivalences of $\mathbf{i}$ )-iv) are established in [2], as is the (trivial) verification that $C_{\varphi}$ is weak* -weak continuous. The equivalences between $\mathbf{i}^{\prime}$ )-iii') follow from Gantmacher's theorem. We hence only need to show that i') implies iv), which we do by following the proof of i) implies iv) and applying the criterion given by Corollary 3.3.

If iv) does not hold, there is a sequence $\left(z_{n}\right)$ in $\mathbb{D}$ converging to a point $z_{0} \in \partial \mathbb{D}$ such that $w\left(z_{n}\right) \geq \operatorname{cv}\left(\varphi\left(z_{n}\right)\right)$ for all $n$, for some $c>0$. Since $v$ is essential, we can choose $f_{n}$ such that $\left\|f_{n}\right\|_{H_{v}^{\infty}}=1$ and $\left|f_{n}\left(\varphi\left(z_{n}\right)\right)\right| \sim 1 / v\left(\varphi\left(z_{n}\right)\right)$. It has to hold that $\left|\varphi\left(z_{n}\right)\right| \rightarrow 1$, or i') would be contradicted; we may select non-negative integers $\alpha_{n} \rightarrow \infty$ such that $\left|\varphi\left(z_{n}\right)\right|^{\alpha_{n}} \geq 1 / 2$ for all $n$. Consider the functions $g_{n}=z^{\alpha_{n}} f_{n}$. Since $|z|^{\alpha_{n}}$ tends pointwise to zero in $\mathbb{D}$, and $\left|f_{n}\right| v_{n}$ is uniformly bounded, it follows by dominated convergence that $g_{n}$ converges to zero in $X=$ $L_{a}^{2}\left(v^{2}\right)$. However,

$$
\left\|C_{\varphi} g_{n}\right\|_{H_{w}^{\infty}} \geq\left|g_{n}\left(\varphi\left(z_{n}\right)\right) w\left(z_{n}\right)\right| \geq c\left|\varphi\left(z_{n}\right)\right|^{\alpha_{n}}\left|f_{n}\left(\varphi\left(z_{n}\right)\right)\right| v\left(\varphi\left(z_{n}\right)\right) \gtrsim \frac{c}{2},
$$

contradicting (7).

For the next examples we introduce the spaces BMOA and VMOA of analytic functions of bounded and vanishing mean oscillation on the unit disc $\mathbb{D}$. To fit them into our framework, for $a \in \mathbb{D}$ and $\lambda \in \mathbb{T}$, let $\phi_{a, \lambda}$ be the disc automorphism

$$
\phi_{a, \lambda}(z)=\lambda \frac{a-z}{1-\bar{a} z} .
$$

Further, let $X=Y=H^{2} / \mathbb{C}$, where $H^{2}$ is the usual Hardy space on the disc, and let $\mathcal{L}$ consist of all composition operators $L_{\phi_{a, \lambda}}: H^{2} / \mathbb{C} \rightarrow H^{2} / \mathbb{C}$,

$$
L_{\phi_{a, \lambda}} f=f \circ \phi_{a, \lambda}-f\left(\phi_{a, \lambda}(0)\right) .
$$

We equip $\mathcal{L}$ with the topology of $\mathbb{D} \times \mathbb{T}$. Then

$$
M\left(H^{2} / \mathbb{C}, \mathcal{L}\right)=\text { BMOA }, \quad M_{0}\left(H^{2} / \mathbb{C}, \mathcal{L}\right)=\mathrm{VMOA},
$$


see ([14], Example 4.2). In a similar fashion we also obtain the Bloch spaces $B$ and $B_{0}$,

$$
M\left(L_{a}^{2} / \mathbb{C}, \mathcal{L}\right)=B, \quad M_{0}\left(L_{a}^{2} / \mathbb{C}, \mathcal{L}\right)=B_{0},
$$

by letting $X=Y=L_{a}^{2} / \mathbb{C}=\left(L^{2}(\mathbb{D}) \cap \operatorname{Hol}(\mathbb{D})\right) / \mathbb{C}$ be the standard analytic Bergman space on the disc (modulo constants) and letting $\mathcal{L}$ be the same collection of composition operators.

Example 3. Let $\varphi: \mathbb{D} \rightarrow \mathbb{D}$ be an analytic function. Several concrete realizations of Example 1 can be given by considering composition operators $C_{\varphi}$ acting on spaces of analytic functions. In [17] it is shown that $C_{\varphi}: Z \rightarrow B$, where $Z=B$ or $Z=\mathrm{BMOA}$, is compact if and only if $\lim _{|a| \rightarrow 1}\left\|C_{\varphi} \phi_{a, \lambda}\right\|_{B}=0$, yielding that $C_{\varphi}: Z \rightarrow B$ is weakly compact if and only if compact. If $\varphi \in B_{0}$, then $C_{\varphi}$ acts boundedly on $B_{0}$, and it follows in combination with Gantmacher's theorem that $C_{\varphi}: B_{0} \rightarrow B_{0}$ is weakly compact if and only if compact, a result first shown in [13].

In connection with Example 3 we also mention the work of Laitila et. al. [8], where it was shown that $C_{\varphi}: \mathrm{BMOA} \rightarrow \mathrm{BMOA}$ is (weakly) compact if and only if $\lim _{|a| \rightarrow 1}\left\|C_{\varphi} \phi_{a, \lambda}\right\|_{\mathrm{BMOA}}=$ 0 . A version of Corollary 3.3 for $M=Z=\mathrm{BMOA}$ and $T=C_{\varphi}$ actually appears as an ingredient of their paper. This is in line with other recent contributions to the field of compact composition operators, e.g. [3] and [11], where the use of Banach space techniques has been essential. In fact, something reminiscent of Theorem 3.1 plays an important role in all of the cited articles.

We conclude with an example of integral operators. The symbols of the operators will belong either to the logarithmic BMOA-space LMOA $=M\left(H^{2} / \mathbb{C}, \mathcal{K}\right)$, or its corresponding small space $\mathrm{LMOA}_{0}=M_{0}\left(H^{2} / \mathbb{C}, \mathcal{K}\right)$. Here we have chosen $X=Y=H^{2} / \mathbb{C}$ and the collection $\mathcal{K}$ of operators to consist of the weighted compositions

$$
K_{\phi_{a, \lambda}} f=\log \frac{2}{1-|a|}\left[f \circ \phi_{a, \lambda}-f\left(\phi_{a, \lambda}(0)\right)\right] .
$$

Example 4. For an analytic function $g$ in $\mathbb{D}$, we denote by $T_{g}$ the Volterra-type operator

$$
T_{g} f(z)=\int_{0}^{z} f(\zeta) g^{\prime}(\zeta) d \zeta, \quad z \in \mathbb{D}
$$

acting on analytic functions $f$ in $\mathbb{D}$. Siskakis and Zhao $[\mathbf{1 6}]$ showed that $T_{g}:$ BMOA $\rightarrow$ BMOA is bounded if and only if $g \in \mathrm{LMOA}$. They proved in the same paper that $T_{g}: \mathrm{BMOA} \rightarrow \mathrm{BMOA}$ is compact if and only if $g \in \mathrm{LMOA}_{0}$, and posed the question whether $T_{g}: \mathrm{BMOA} \rightarrow \mathrm{BMOA}$ can be weakly compact without being compact. This was answered in the negative by Laitila, Mihkinen, and Nieminen [7]. The purpose of this example is to illustrate that the question may in fact be resolved using Siskakis and Zhao's original argument, when applied in conjuction with Corollary 3.3.

First we point out that the boundedness of $T_{g}, g \in$ LMOA, automatically implies that $T_{g}(\mathrm{VMOA}) \subset \mathrm{VMOA}$, so that $\left.T_{g}\right|_{\mathrm{VMOA}}: \mathrm{VMOA} \rightarrow \mathrm{VMOA}$ is a bounded operator. Secondly, it is easily verified that $\left(\left.T_{g}\right|_{\mathrm{VMOA}}\right)^{* *}=T_{g}$. That is, $T_{g}$ is weak ${ }^{*}$-weak continuous. By Gantmacher's theorem it follows that $T_{g}$ (or equivalently $\left.T_{g}\right|_{\mathrm{VMOA}}$ ) is weakly compact if and only if $T_{g}(\mathrm{BMOA}) \subset \mathrm{VMOA}$.

When proving that compactness implies $g \in \mathrm{LMOA}_{0}$ in [16], the only step where compactness is used, as opposed to weak compactness, is in showing that $\lim _{n}\left\|T_{g} q_{n}\right\|_{\mathrm{BMOA}}=0$, where

$$
q_{n}(z)=\log \frac{1-\bar{u} z}{1-\bar{u}_{n} z}
$$


for a point $u \in \partial \mathbb{D}$ and a sequence $\left(u_{n}\right) \subset \mathbb{D}$ of points converging to $u$. However, $q_{n}$ is uniformly bounded in BMOA,

$$
\left\|q_{n}\right\|_{\mathrm{BMOA}} \lesssim\|\log (1-z)\|_{\mathrm{BMOA}}
$$

and $\lim _{n}\left\|q_{n}\right\|_{H^{2}}=0$, so it follows from Corollary 3.3 that $T_{g} q_{n} \rightarrow 0$ in BMOA, assuming only the weak compactness of $T_{g}$. With this remark in hand, one can follow the proof in [16] verbatim to see that $T_{g}$ is weakly compact if and only if $g \in \mathrm{LMOA}_{0}$.

Acknowledgements. The author is grateful to the referee for valuable comments that helped to improve the paper.

\title{
References
}

1. Bessaga, C. and Pefczyński, A., On bases and unconditional convergence of series in Banach spaces, Studia Math. 17 (1958), 151-164.

2. Bonet, J., Domański, P., Lindström, M., Taskinen, J., Composition operators between weighted Banach spaces of analytic functions, J. Austral. Math. Soc. Ser. A 64 (1998), 101-118.

3. Contreras, M. D., Diaz-Madrigal, S. and Vukotic D., Compact and weakly compact composition operators from the Bloch space into Möbius invariant spaces, J. Math. Anal. Appl. 415 (2014), 713-735.

4. Diestel, J., Sequences and series in Banach spaces, Graduate Texts in Mathematics 92, Springer-Verlag, New York, 1984

5. Harmand, P., Werner, D., Werner, W., M-ideals in Banach spaces and Banach algebras, Lecture Notes in Mathematics 154, Springer-Verlag, Berlin, 1993.

6. JaRchow, H., On weakly compact operators on C-algebras, Math. Ann. 273 (1986), 341-343.

7. Laitila, J., Minhkinen, S. and Nieminen, P. J., Essential norms and weak compactness of integration operators, Arch. Math. (Basel) 97 (2011), 39-48.

8. Laitila, J., Nieminen, P. J., Saksman, E. and Tylli, H.-O., Compact and weakly compact composition operators on BMOA, Complex Anal. Oper. Theory 7 (2013), 163-181.

9. Lefìvre, P., Some characterizations of weakly compact operators in $H^{\infty}$ and on the disk algebra. Application to composition operators, J. Operator Theory 54 (2005), 229-238.

10. Lefèvre, P., Li, D., Queffélec, H. and Rodríguez-Piazza, L., A criterion of weak compactness for operators on subspaces of Orlicz spaces, J. Funct. Spaces Appl. 6 (2008), 277-292.

11. Lefèvre, P., Li, D., Queffélec, H. and Rodríguez-Piazza, L., Composition operators on Hardy-Orlicz spaces, Mem. Amer. Math. Soc. 207 (2010).

12. LеĬвоv, M. V., Subspaces of the space VMO, (Russian) Teor. Funktsii Funktsional. Anal. i Prilozhen 46 (1986), 51-54; translation in J. Soviet Math. 48 (1990), 536-538

13. Madigan, K., Matheson, A., Compact composition operators on the Bloch space, Trans. Amer. Math. Soc. 347 (1995), 2679-2687.

14. Perfekt, K.-M., Duality and distance formulas in spaces defined by means of oscillation. Ark. Mat. 51 (2013), 345-361.

15. Perfekt, K.-M., On M-ideals and o-O type spaces, preprint (2014), arXiv:1412.5486.

16. Siskakis, A. G. and ZhaO, R., A Volterra type operator on spaces of analytic functions, Function spaces (Edwardsville, IL, 1998), 299-311, Contemp. Math., 232, Amer. Math. Soc., Providence, RI, 1999.

17. TJani, M., Compact composition operators on some Moebius invariant Banach spaces, Thesis (Ph.D.), Michigan State University, 1996.

\author{
K.-M. Perfekt \\ Department of Mathematical Sciences \\ Norwegian University of Science and \\ Technology \\ 7491 Trondheim \\ Norway \\ karl-mikael.perfekt@math.ntnu.no
}

